\title{
Self-efficacy impact adherence in diabetes mellitus
}

\author{
D'Souza MS ${ }^{1 *}$ and Al Salmi NMD ${ }^{2}$ \\ ${ }^{1}$ Abbotsford Regional Hospital and Cancer Centre, British Columbia V2T 1S3, Canada \\ ${ }^{2}$ AG-CNS, College of Nursing, Sultan Qaboos University, Muscat, Oman
}

\begin{abstract}
Aim: The aim of the paper is to explore how self-efficacy is associated with adherence among adults with DM.

Methods: The search of electronic databases identified 564 records from 2007 to 2017 on self-efficacy and adherence from different perspectives and its effect on adults with DM.

Discussion: Self-efficacy increases the confidence in adults in their self-care behaviours. Non-adherence continues to be a significant barrier to self-efficacy. Selfefficacy and adherence should be informed by an understanding of theoretical frameworks and the individual characteristics.

Conclusion: Adherence is likely among adults with better self-efficacy to empower them to make valid decisions about their health. Interventions to improve selfefficacy should be tailored based on different types of non-adherence such as intentional and unintentional non-adherence.
\end{abstract}

Implications: An inter-collaborative professional practice approach is crucial to improve self-efficacy and adherence for sound judgment and valid decision making.

\section{Introduction}

Three hundred forty-seven million persons have diabetes mellitus (DM) and nearly 3.4 million persons die from complications [1]. Adults with DM have a high incidence of morbidity and mortality as compared to normal or near-normal adult populations. Adults with DM is expected to rise to 350 million cases by the year 2030 [2]. In developed countries, non-adherence to the treatment of chronic diseases ranges from $30 \%$ to $50 \%$, and this rate has escalated in the developing countries [3]. Lifestyle factors like behavioural, environmental and societal play a critical part in the prevention of both development of the disease and its complications [4].

Adults with DM are at higher risk of developing cardiovascular and other secondary complications if the DM is not well controlled [5]. Poor adherence affects self-efficacy leading to lower quality of life and imposes a significant financial burden on the healthcare [6]. Poor adherence and lack of knowledge are key concerns of developing complications and to managing DM. In adults with DM requiring behavior change, self-efficacy and self-care behaviours are central to adherence. Non-adherence to prescribed medications and the lack of awareness are believed to be the common causes of poor treatment adherence among Arab adults.

The Middle Eastern countries will have the second highest increase in the percentage of people with DM in 2030 compared to other parts of the world [7]. In the Sultanate of Oman, approximately $10 \%$ of the population is currently living with DM. The number of adults with DM in Oman will rise from 75,000 in 2000 to 217,000 in 2025 [8]. There are very few studies on self-efficacy and the relationship between sociodemographic characteristics and adherence levels of adults with DM in the Middle East [6]. The aim of the paper is to explore how self-efficacy is associated with adherence among adults with DM.

\section{Methods}

Several research databases (Academic Search, CINAH, EBSCO, Google Scholar, JSTOR, Lippincott Williams and Wilkins, Medline, ProQuest, PsycINFO, Sage, Science Direct, Springer, Web of Science, and Wiley) were reviewed with keywords for self-efficacy, self-care behaviours, adherence, compliance, chronic illness, adults, and diabetes mellitus. The search of electronic databases identified 564 records from 2007 to 2017 on self-efficacy and adherence from different perspectives and its effect on adults with DM.

\section{Review}

Adherence is defined as, an extent to which a person's behaviortaking medication, following a diet, and or executing lifestyle changes; corresponds with the agreed recommendations from a provider [9]. The concept of adherence implies a mutual and dynamic interaction between the patients and health care providers, and it results in longterm changes in patients' behaviours [3]. The barriers to adherence are poor knowledge, psycho-social status, the complexity of the disease, co-morbidity, lack of comprehension of treatment, and poor communication between the patient and health-care provider [10].

A qualitative study to explore the possibility of applying treatment adherence success factors in diabetes consultations between health care providers and patients [11]. Patients did not experience goalsetting in consultations. Healthcare providers indicated they motivated patients to set treatment goals. Though shared-decision making was

${ }^{\star}$ Correspondence to: Melba Sheila D’Souza, RN, Abbotsford Regional Hospital and Cancer Centre, British Columbia V2T 1S3, Canada, E-mail: melba123@rediffmal.com

Received: October 19, 2018; Accepted: November 02, 2018; Published: November 07, 2018 
applied, patients were passive collaborators and healthcare providers were in charge of making treatment decisions. An effect of educational interventions on knowledge of the disease, treatment adherence, and control of diabetes mellitus used a pre- and post-test design with the single comparison group [12]. Educational intervention using the cognitive social theory had significantly improved the medication treatment adherence.

An investigation of the DM medication adherence using a theoretical framework, the Health Belief Model (HBM) was conducted in Saudi Arabia [13]. The regression analyses found that most of the adults took the prescribed dose every time taken; however, $60 \%$ of these adults were not taking the dose in the prescribed number of times per day and $50 \%$, were not taking the medication in the prescribed time of the day. Perceived susceptibility, perceived medication benefits, and self-efficacy were significant HBM predictors for medication adherence $(\mathrm{R} 2=0.42)[13]$.

Self-reported adherence rate to anti-diabetic drugs was $84 \%$ and the most common reason for non-adherence was forgetfulness in the United Arab Emirates [14]. This strategy is highly influenced by social desirability responding (faking good), and it depends on memory limitations, especially when asking about behaviours that required adults to remember old practices [15]. Medication adherence of adults with DM in Oman was used to identify the probable reasons for medication non-adherence [6]. Forgetfulness was the most frequent reason for medication non-adherence (36.4\%) [6].

Developing self-efficacy and self-care behaviours are complex, difficult to incorporate into lifestyles and is influenced by a myriad of psych-social factors like motivation, adherence, compliance etc. Adults with DM are responsible for commitment, new skills, knowledge, confidence, compliance, and adherence. It involves a more detailed assessment to provoke changes in self-efficacy for better adherence and understanding of the social and behavioural theories like Social Cognitive Theory, Health Belief Model, Theory of Reasoned Action, and Theory of Planned Behaviour. Social cognitive theory addresses both self-efficacy and outcome expectations which are motivating factors [16]. This theory states that when people observe a model performing a behavior and the consequences of that behavior, they remember the sequence of events and use this information to guide subsequent behaviours.

Self-efficacy refers to people's beliefs about their capabilities to produce designated levels of performance that can influence events which affect their lives. Self-efficacy as the 'belief in one's capabilities to organize and execute the sources of action required to manage prospective situations' [17]. Self-efficacy is the central concept that determines individuals' behaviours and how much effort they spend on adopting the behavior. The perceptions of managing psychosocial aspects of diabetes and readiness to change affect goal attainment in the empowerment model [18]. Higher ability to manage diabetes positively significantly predicts the quality of life and body mass index in the health-related quality of life model [19]. It is significant to recognize paradigms that are pertinent at different phases of readiness for change that are beneficial for self-efficacy. Self-efficacy is the belief that one can effectively achieve the behavior essential to adherence.

Self-efficacy played a critical mediating role between symptoms of depression- a common co-morbidity with DM and glycemic control. Predictors of self-efficacy (SE) are demographic and clinical characteristics accounting of $20.6 \%$ of the total variance in self-care behaviours and $31.3 \%$ of the variance of the SE in the self-efficacy model [20]. Boosting adults' self-efficacy (confidence) with regard to their ability to implement care successfully is a critical step in promoting active self-management [21]. Behavior change requires some 'intention' and 'intention' is driven by 3 constructs: attitude, subjective norm, and self-efficacy [22]. Self-efficacy is an important component of management and a key psychosocial variable used in predicting adherence to self - care behaviours.

Central to the theories is the construct of self-efficacy and selfcare behaviours and how these influence adherence. Higher selfefficacy is linked with SCB in determining glycemic control and lower HbA1c levels [23]. Education, understanding of DM and management predicted good foot care behaviours in the self-efficacy model [24]. Selfefficacy is the most powerful determinant of intention and suggested attention to self-efficacy. The relationship between these is critical to the improved self-efficacy and adherence among adults with DM.

\section{Discussion}

Exploring self-efficacy is critical to achieving optimal adherence among adults with DM. Self-efficacy increases the confidence in adults in their self-care behaviours. [25] The common applications are of social cognitive theory within which the health belief model, the theory of reasoned action, and the theory of planned behavior are most prevalent. The majority of the reviewed studies examining non-adherence have considered it as a single entity and have not differentiated types of non-adherence such as intentional and unintentional non-adherence or the reasons underlying each type. It is very significant to assess before intervene, intentional non-adherence behaviours should be treated differently. Unintentional behaviours such as forgetfulness should receive other strategies to enhance treatment adherence. Nonadherence continues to be a significant barrier to self-efficacy.

Non-adherence concerns all aspects of therapy; not only taking medicines, but also developing self-efficacy and lifestyle changes such as diet and physical exercise, avoiding high-risk behaviours, such as the use of tobacco or alcohol, or simply returning for the next medical appointment. Adults have variant levels in adherence to prescribed treatments. Therefore, the application of theory-driven, evidence-based models is important in the development of effective interventions. Self-efficacy and adherence, should be informed by an understanding of theoretical frameworks (e.g., socio-cognitive, self-regulation, and social support), and within those a range of subordinate models (e.g., health belief model, theory of planned behavior, and self-regulation model) and then the individual characteristics (e.g., perceived barriers, perceived benefits, and treatment beliefs).

With aging and baby boomers, demographical and urbanization changes in society, information motivation behavioural skills, it is shown that self-efficacy impacts adherences as shown in the Attachment theory, Epidemiological theory, Theory of reasoned action, Cognitive behavioural theory, Self-care model impacts adherence, and the Medication adherence model. The interventions to improve self-efficacy should be tailored based on the socio-demographic, psychosocial, behavioural, and lifestyle characteristics, the different types of nonadherence such as intentional (e.g., not attending appointments) and unintentional (e.g., forgetfulness) non-adherence of the targeted population.

\section{Conclusion}

Adherence is likely among adults with better self-efficacy to empower them to make valid decisions about their health. The socio-cultural aspects of adults in the Middle East are different from 
the western world in terms of culture, religious nature, spirituality, beliefs, values, education, awareness level, efficacy, compliance and health practices. Providing explanations and predictions about the self-efficacy phenomenon will help to identify barriers and challenges concerning the adherence interpretation. Empirically, cross-sectional descriptive studies guided by behavioural frameworks and self-efficacy models are needed. Building a good knowledge base with clinical trials and behavioural change interventions can be used to test effective interventions that can improve self-efficacy. Health care professionals need to be trained on how to use cognitive behavioural therapies in their communication and consultation with adults with DM. Nurses can make an important contribution to improving self-efficacy among adults with DM. Hence, treatment adherence is a complex and multidimensional phenomenon and is very important to address the concept from self-efficacy. An inter-collaborative professional practice approach is crucial to improve self-efficacy and adherence for sound judgment and valid decision making.

\section{References}

1. World Health Organization (2016) Global report on diabetes.

2. Guariguata L, Whiting DR, Hambleton I, Beagley J, Linnenkamp U, et al. (2014) Global estimates of diabetes prevalence for 2013 and projections for 2035. Diabetes Res Clin Pract 103: 137-149. [Crossref]

3. Costa E, Giardini A, Savin M, Menditto E, Lehane E, et al. (2015) Interventional tools to improve medication adherence: review of the literature. Patient preference and adherence 9: 1303-1314. [Crossref]

4. Didarloo A, Alizadeh M (2016) Health-related quality of life and its determinants among women with diabetes mellitus: A cross-sectional analysis. Nurs Midwifery Stud 5. [Crossref]

5. Mooradian AD (2003) Cardiovascular disease in DM Mellitus: Current management guidelines. Arch Intern Med 163: 33-40. [Crossref]

6. Jimmy B, Jose J, Al-Hinai ZA, Wadair IK, Al-Amri GH (2014) Adherence to medications among $\mathrm{dm}$ mellitus patients in three districts of al dakhliyah governorate, Oman: A cross-sectional pilot study. Sultan Qaboos Univ Med J 14: e231-e235. [Crossref]

7. Shaw JE, Sicree RA, Zimmet PZ (2010) Global estimates of the prevalence of diabetes for 2010 and 2030. Diabetes Res Clin Pract 87: 4-14. [Crossref]

8. Nazmi A, Khan S, Hadithi D (2013) Self-monitoring of blood glucose level among diabetic patients in Muscat, Oman: A pilot study. Saudi Journal for Health Sciences 2: 54-57.

9. Holmes EA, Hughes DA, Morrison VL (2014) Predicting adherence to medications using health psychology theories: A systematic review of 20 years of empirical research. Value Health 17: 863-876. [Crossref]
10. Reach G (2008) A novel conceptual framework for understanding the mechanism of adherence to long-term therapies. Patient Prefer Adherence 2: 7-19. [Crossref]

11. Vluggen S, Hoving C, Schaper NC, de Vries H (2018) Exploring beliefs on diabetes treatment adherence among Dutch type 2 diabetes patients and healthcare providers. Patient Educ Couns 101: 92-98. [Crossref]

12. Figueira ALG, Boas LCGV, Coelho ACM, Freitas MCF, Pace AE (2017) Educational interventions for knowledge on the disease, treatment adherence, and control of diabetes mellitus. Rev Lat Am Enfermagem 25. [Crossref]

13. Alatawi YM, Kavookjian J, Ekong G, Alrayees MM (2016) The association between health beliefs and medication adherence among patients with DM. Res Social Adm Pharm 12: 914-925. [Crossref]

14. Arifulla M, John LJ, Sreedharan J, Muttappallymyalil J, Basha SA (2014). Patients' adherence to anti-diabetic medications in a hospital at ajman, UAE. Malays $J$ Med SCi 21: 44-49. [Crossref]

15. Levensky ER, O’Donohue WT (2006) Promoting treatment adherence: A practical handbook for health care providers. Thousand oaks, Calif: SAGE Publications, Inc.

16. Nouwen A, Urquhart Law G, Hussain S, McGovern S, Napier H (2009) Comparison of the role of self-efficacy and illness representations in relation to dietary self-care and diabetes distress in adolescents with type 1 diabetes. Psychol Health 24: 1071-1084. [Crossref]

17. Bandura A (1995) Self-efficacy in changing societies. Cambridge university press.

18. D’Souza MS, Karkada SN, Hanrahan NP, Venkatesaperumal R, Amirtharaj A (2015) Do perceptions of empowerment affect glycemic control and self-care among adults with DM? Glob J Health Sci 7: 80-90. [Crossref]

19. D'Souza MS, Venkatesaperumal R, Ruppert SD, Karkada SN, Jacob D (2016) Health related quality of life among omani men and women with type 2 diabetes. J Diabetes Res 2016: 8293579. [Crossref]

20. D'Souza MS, Karkada SN, Parahoo K, Venkatesaperumal R, Achora S, et al. (2017) Self-efficacy and self-care behaviours among adults with type 2 diabetes. Appl Nurs Res 36: 25-32. [Crossref]

21. Wu SF, Huang YC, Lee MC, Wang TJ, Tung HH, et al. (2013) Self-efficacy, self-care behavior, anxiety, and depression in Taiwanese with type 2 diabetes: A cross-sectional survey. Nurs Health Sci 15: 213-219. [Crossref]

22. Didarloo, A., Shojaeizadeh, D, Alizadeh, M (2016b) Impact of educational intervention based on interactive approaches to beliefs, behavior, hemoglobin A1c, and quality of life in diabetic women. Int J Prev Med 7. [Crossref]

23. D'Souza MS, Karkada SN, Venkatesaperumal R, Natarajan J (2015) Self-care behaviors and glycemic control among adults with DM. GSTF Journal of Nursing and Health Care 2.

24. D'Souza MS, Ruppert SD, Parahoo K, Karkada SN, Amirtharaj A, et al. (2016) Foo care behaviors among adults with type 2 diabetes. Prim Care Diabetes 10: 442-451. [Crossref]

25. Doggrell SA, Warot S (2014) The association between the measurement of adherence to anti-diabetes medicine and the HbA1c. Int J Clin Pharm 36: 488-497. [Crossref]

Copyright: (C2018 D’Souza MS. This is an open-access article distributed under the terms of the Creative Commons Attribution License, which permits unrestricted use, distribution, and reproduction in any medium, provided the original author and source are credited. 